\title{
OPTIMAL SWITCHING IN AN ECONOMIC ACTIVITY UNDER UNCERTAINTY
}

Kjell Arne Brekke ${ }^{1)}$ and Bernt Øksendal 2)

1) Central Bureau of Statistics

Box 8131 Dep

N-0033 Oslo 1

Norway

2) Department of Mathematics

University of Oslo

Box 1053, Blindern

N-0316 Oslo 3

Norway

\begin{abstract}
We consider the problem of finding the optimal sequence of opening (starting) and closing (stopping) times of a multi-activity production process, given the costs of opening, running and closing the activities, and assuming that the state of the economic system is a stochastic process. The problem is formulated as an extended impulse control problem and solved using stochastic calculus.
\end{abstract}

As an application we find explicitly the optimal starting and stopping strategy for a resource extraction when the price of the resource is following a geometric Brownian motion. 


\title{
OPTIMAL SWITCHING IN AN ECONOMIC \\ ACTIVITY UNDER UNCERTAINTY
}

\author{
Kjell Arne Brekke and Bernt Øksendal
}

\section{§1. Introduction}

Optimal stopping has a wide variety of applications in economics, ranging over real and financial option, entry to a market or optimal start of a production process under uncertainty. But in many applications it will also be natural to consider the possibility of the reverse action, like exiting from a market or shutting down a production. For example, there are industries where part of the production process is temporarily shut down when electricity prices are too high; at high prices all workers are relocated to other tasks and when the prices fall below a certain limit production is restarted.

The purpose of this paper is to extend the results of $[\mathrm{B} \varnothing]$ and to give an affirmative answer to a question left open there. More precisely, we give sufficient conditions in terms of quasi-variational inequalities that a given function actually is the maximal expected profit function and we describe the corresponding optimal starting and stopping strategy. For concreteness the results are applied to the following problem of optimal starting and stopping of a resource depletion with a stochastic price development:

Suppose it costs the amount $L$ to open a field for resource extraction, that the running/rental cost is $K$ per time unit and that the cost of closing down a field is $C$. If the price of the resource in consideration is varying as a stochastic process (to be specified below), when is the optimal time to open the field and to close it? It seems reasonable that if the field is open, it may be a good strategy to continue the extraction for a while even if the price has gone below the running costs, because there may be a chance that prices could go up again and closing and re-opening the field is costly. On the other hand, even with such an optimistic prospect there is clearly a limit as to how low the prices can go before closing is the optimal strategy. Similarly, if the field is closed one would wait for a resource price which is higher than the running costs before opening again. But how high? The purpose of this paper is to formulate this problem mathematically in terms of impuls control and solve the problem explicity using stochastic calculus.

The starting and stopping problem has been considered in various contexts. It was discussed in connection with taxes and convenience yield by Brennan and Schwartz [BS]. A similar entry and exit model (but without resource extraction) has been studied by Dixit [D]. Neither of these papers give a rigorous mathematical proof that an optimal starting and stopping strategy exists and that it has the form stated. The more general problem of starting and stopping several activities simultaneously is considered in [MZ], in the context of oil exploration.

In $[\mathrm{B} \varnothing]$ a candidate $\phi_{0}$ for the solution of the resource extraction problem is found explicitly, as an application of a high contact principle for optimal stopping. But it is not proved there that this candidate actually is the solution. This will be established in this paper. More generally, we consider the problem of optimal starting and stopping of a multi- 
activity system under uncertainty. We prove that a given function satisfying certain quasivariational inequalities necessarily is the solution of the problem.

This paper is organized as follows: In §2 we formulate a general starting and stopping problem as an impulse control problem. In $\S 3$ we give sufficient conditions that a given function and its associated starting and stopping strategy solves the general problem in $\S 2$. Then in $\S 4$ we apply this to the specific problem of optimal resource extraction mentioned above.

\section{\$2. A mathematical formulation of the problem}

The problems mentioned in the introduction are special cases of the following general problem:

Suppose there are $m$ possible "indicator values" $z_{1}, \cdots, z_{m}$ of the state of the system at time $t$. Let $Z_{t}$ denote the value of this indicator at time $t$, so that for all $t$

$$
Z_{t} \in\left[z_{1}, \cdots, z_{m}\right\}=: \mathcal{Z}
$$

Remark. If, for example, we consider a firm with $k$ production activities which can be either "on/open" or "off/closed", then each indicator value $z \in \mathcal{Z}$ can be represented as a $k$-tuple

$$
z=\left(a_{1}, a_{2}, \cdots, a_{k}\right)
$$

where each $a_{i}$ is either 0 (meaning activity $\mathrm{nr}$. $i$ is closed) or 1 (meaning activity $\mathrm{nr}$. $i$ is open). So in this case there are $m=2^{k}$ possible indicator values.In particular, in the resource extraction case there are just 2 indicator values which we denote by 0 or 1 depending on whether the field is closed or open.

The firm's environment at time $t$, e.g. prices of output or input goods, is denoted by $U_{t}$. We assume that $U_{t}$ is a stochastic process in $\mathbf{R}^{n}$ satisfying the following stochastic differential equation

$$
d U_{t}=b\left(t, U_{t}, Z_{t}\right) d t+\sigma\left(t, U_{t}, Z_{t}\right) d B_{t}
$$

where $b: \mathbf{R}^{n+1} \times \mathcal{Z} \rightarrow \mathbf{R}^{n}, \sigma: \mathbf{R}^{n+1} \times \mathcal{Z} \rightarrow \mathbf{R}^{n \times m}$ are Lipschitz functions in the first $n+1$ variables and $B_{t}$ denotes $m$-dimensional Brownian motion. (See e.g. [Ø] for more information on stochastic differential equations.)

The state of the whole economic system at time $t$ is represented by the stochastic process

$$
X_{t}=\left[\begin{array}{c}
t \\
U_{t} \\
Z_{t}
\end{array}\right]
$$

The probability law of $X_{t}$ given that $X_{0}=x=(t, u, z)$ is denoted by $P^{x}$ and expectation with respect to $P^{x}$ is denoted by $E^{x}$.

An impulse control $w$ for this system consists of a double (possibly finite) sequence

$$
w=\left(\theta_{1}, \theta_{2}, \cdots, \theta_{k}, \cdots ; \zeta_{1}, \zeta_{2}, \cdots, \zeta_{k}, \cdots\right)_{k \leq N} \quad(N \leq \infty)
$$


where each $\theta_{k} \leq \infty$ is a stopping time (with respect to the filtration $\left\{\mathcal{F}_{t}\right\}$ for the Brownian motion $\left\{B_{t}\right\}$ ), $\theta_{k} \leq \theta_{k+1}$ and $\theta_{k} \rightarrow \infty$ a.s. (so if $N$ is finite then $\theta_{N} \equiv \infty$ ). Associated to the impuls time $\theta_{k}$ is the impulse $\zeta_{k} \in \mathcal{Z}$ which determines the new value of $Z_{t}$ at time $t=\theta_{k}$.

We may regard $\theta_{1}, \theta_{2}, \cdots$ as the times when we decide to interfere with the system and the corresponding $\zeta_{1}, \zeta_{2} \cdots$ are the new indicator values that we give the system at these times. We often simplify the notation and write $w=\left(\theta_{1}, \theta_{2}, \cdots\right)$. Let $W$ denote the set of all impulse controls.

If $w \in W$ is applied to the system it gets the form

$$
X_{t}=X_{t}^{(w)}=\left[\begin{array}{c}
t \\
U_{t} \\
\zeta_{k}
\end{array}\right] \quad \text { if } \theta_{k} \leq t<\theta_{k+1} .
$$

Note that $X_{t}^{(w)}$ is right-continuous for all $w \in W$. Let $E^{x}$ denote the expected value when $X_{0}=x=(t, u, z)$.

Let $f(x)$ denote the profit per time unit when the system is in the state $x$. For $x=$ $(t, u, z) \in \mathbf{R}^{n+1} \times \mathcal{Z}$ and $\zeta \in \mathcal{Z}$ let $H(x, \zeta) \in \mathbf{R}$ be the cost of switching the indicator value from $z$ to $\zeta$ when the state is $x=(t, u, z)$. Then the expected total profit of running the system with the impulse control $w=\left(\theta_{1}, \theta_{2}, \cdots ; \zeta_{1}, \zeta_{2}, \cdots\right) \in W$ is given by

$$
J^{w}(x)=E^{x}\left[\int_{t}^{\infty} f\left(X_{s}^{(w)}\right) d s-\sum_{j=1}^{\infty} H\left(X_{\theta_{j}-}, \zeta_{j}\right)\right]
$$

where $X_{\theta_{j-}}=\lim _{t \dagger \theta_{j}} X_{t}$.

We assume that the switching cost function $H: \mathbf{R}^{n+1} \times \mathcal{Z} \times \mathcal{Z} \rightarrow \mathbf{R}^{+}$satisfies

$$
H(x, \zeta)>0 \text { for all } x \in \mathbf{R}^{n+1} \times \mathcal{Z} \text { and all } \zeta \neq z
$$

and - if $\mathcal{Z}$ consists of more than 2 elements -

$$
H\left(t, u, z, \zeta_{2}\right) \leq H\left(t, u, z, \zeta_{1}\right)+H\left(t, u, \zeta_{1}, \zeta_{2}\right) \text { if } z \neq \zeta_{1} \neq \zeta_{2} \neq z .
$$

We also assume that

$$
(t, u) \rightarrow H(t, u, z, \zeta) \text { is continuous for all } z, \zeta \text {. }
$$

(the values of $H$ when $z=\zeta$ are not used, so we only need to define $H(t, u, z, \zeta)$ for $z \neq \zeta$ ).

\section{Remarks.}

(i) Condition (2.8) states that if we want to switch from indicator state $z$ to indicator state $\zeta_{2}$, then it is not more expensive to do this directly (in one step) than in two steps, via an intermediate indicator value $\zeta_{1}$. For example, if

$$
H(t, u, z, \zeta)=e^{-\rho t} H_{0}(z, \zeta) \quad(\rho \text { constant }) .
$$


then (2.8) becomes the 'triangle inequality'

$$
H_{0}\left(z, \zeta_{2}\right) \leq H_{0}\left(z, \zeta_{1}\right)+H_{0}\left(\zeta_{1}, \zeta_{2}\right) \quad z \neq \zeta_{1} \neq \zeta_{2} \neq z
$$

If we are given a function $H$ satisfying (2.7) and (2.9) we can always modify it to satisfy (2.8) as well, by putting

$$
\tilde{H}(t, u, z, \zeta)=H(t, u, z, \zeta) \wedge \min _{z \neq \zeta_{1} \neq \zeta}\left\{H\left(t, u, z, \zeta_{1}\right)+H\left(t, u, \zeta_{1}, \zeta\right)\right\}
$$

(ii) In the resource extraction example the switching cost function has the values

$$
\begin{aligned}
& H(t, u, 0,1)=L e^{-\rho t} \quad \text { (discounted opening cost) } \\
& H(t, u, 1,0)=C e^{-\rho t} \quad \text { (discounted closing cost), }
\end{aligned}
$$

where $\rho>0$ is a (constant) discounting factor.

We can now formulate the switching problem as follows:

PROBLEM 2.1. Find

$$
\tilde{\phi}(x):=\sup _{w \in W} J^{w}(x)
$$

and find - if possible - an optimal impulse control $\tilde{w}$, i.e. find $\tilde{w} \in W$ such that

$$
\tilde{\phi}(x)=J^{\bar{w}}(x) \text {. }
$$

Remark. This is essentially an impulse control problem of the type considered in [BL]. However, in [BL] it is assumed that $-f$ is positive (or lower bounded), and this is not a reasonable assumption in our economic application. Therefore it is not possible to apply their results directly to our situation. Nevertheless, our method is inspired by their approach.

In $[\mathrm{B} \varnothing]$ a candidate $\phi_{0}(x)$ for the solution of Problem 2.1 in the specific application of starting and stopping a resource extraction (see §4) was found by adopting the following dynamic programming argument: Suppose the system initially is in state $x=(t, u, z)$. Then if at a stopping time $\tau$ we interfere and start/stop the system, the system gets the impuls $\zeta=1-z$ and then the new state becomes $X_{\tau}=\left(\tau, U_{\tau}, Z_{\tau}\right)$, where $Z_{\tau}=\zeta$. The cost of this operation is

$$
H\left(X_{\tau^{-}}, \zeta\right)=H\left(\tau, U_{\tau}, z, 1-z\right)
$$

where $H$ is given by (2.10) above. From then on the maximal profit is $\tilde{\phi}\left(X_{\tau}\right)$. This procedure can of course at most be optimal. We conclude that, for all stopping times $\tau$,

$$
\tilde{\phi}(x) \geq E^{\alpha}\left[\int_{t}^{\tau} f\left(X_{s}\right) d s-H\left(X_{\tau^{-}}, 1-Z_{\tau^{-}}\right)+\tilde{\phi}\left(X_{\tau}\right)\right]
$$

If an optimal impulse control $\tilde{w}=\left(\tilde{\theta}_{1}, \tilde{\theta}_{2}, \cdots\right)$ exists, then by choosing $\tau=\tilde{\theta}_{1}$ we get equality in (2.11). Hence $\tilde{\phi}$ must satisfy the equation

$$
\tilde{\phi}(x)=\sup _{\tau} E^{x}\left[\int_{t}^{\tau} f\left(X_{s}\right) d s-H\left(X_{\tau^{-}}, 1-Z_{\tau^{-}}\right)+\tilde{\phi}\left(X_{\tau}\right)\right]
$$


Using the 'high contact principle' a solution $\phi_{0}(x)$ of equation (2.12) for the resource extraction problem was found in $[\mathrm{B} \emptyset]$. However, this does not prove that $\phi_{0}=\tilde{\phi}$, because there may (a priori) be several solutions of equation (2.12). We will in $\S 4$ prove that we indeed have $\phi_{0}=\tilde{\phi}$ (under certain conditions). This will be obtained as an application of the more general results we develop in $§ 3$.

\section{§3. Solution of the optimal switching problem}

From now on we put, for fixed $z \in \mathcal{Z}$,

$$
Y_{t}=\left(t, U_{t}, z\right) \quad\left(=\left(t, U_{t}\right) \quad \text { if we suppress } z\right)
$$

so that $Y_{t}$ represents the state of the system corresponding to the "non-interference" impulse control $w_{\infty}=\left(\theta_{1}\right)$ where $\theta_{1}=\infty$. Then $Y$ is a diffusion with generator $A$ given by

$$
A=\frac{\partial}{\partial t}+\sum_{i=1}^{n} b_{i} \frac{\partial}{\partial u_{i}}+\frac{1}{2} \sum\left(\sigma \sigma^{T}\right)_{i j} \frac{\partial^{2}}{\partial u_{i} \partial u_{j}}
$$

In the following we suppress the constant $z$ and regard $Y_{t}$ as the $(n+1)$-dimensional process $\left(t, U_{t}\right)$.

The following concept is useful:

DEFINITION 3.1. We say that a function $g(x)$ is stochastically $C^{2}$ in a domain $D C$ $\mathbf{R}^{n+1}$ (with respect to $Y_{t}$ ) if all the first partial derivatives of $g$ with respect to $t$ and $u$ and all the second partial derivatives of $g$ with respect to $u$ exist a.e. in $D$ with respect to the Green measures $G(y, \cdot)$ of $Y_{t}$ and the following generalized Dynkin formula holds:

$$
E^{y}\left[g\left(Y_{\theta^{\prime}}\right) \mid \mathcal{F}_{\theta}\right]=g\left(Y_{\theta}\right)+E^{y}\left[\int_{\theta}^{\theta^{y}} A g\left(Y_{s}\right) d s \mid \mathcal{F}_{\theta}\right]
$$

for all stopping times $\theta \leq \theta^{\prime} \leq \tau_{D}$, where $\mathcal{F}_{\theta}$ is the filtration generated by $\left\{B_{t \wedge \theta}(\cdot)\right\}_{t \geq 0}$,

$$
\tau_{D}=\inf \left\{t>0 ; Y_{t} \notin D\right\}
$$

and we assume $E^{y}\left[\tau_{D}\right]<\infty$.

Remark. The Green measure $G(y, \cdot)$ is defined by

$$
G(y, F)=E^{y}\left[\int_{0}^{T_{D}} \chi_{F}\left(Y_{s}\right) d s\right] ; \quad y \in D, F \quad \text { Borel set in } D
$$

In (3.3) $A g$ is the operator $A$ applied to $g$, which makes sense a.e. $G(y, \cdot)$ and therefore makes sense in (3.3).

By the classical Dynkin formula all $C^{2}$ functions $g$ which satisfy $E^{y}\left[\int_{0}^{\tau}\left|A g\left(Y_{s}\right)\right| d s\right]<\infty$ for all $y$ are stochastically $C^{2}$. In [BØ, Lemma 1] conditions are given which imply that a $C^{1}$ 
function which is $C^{2}$ outside a "thin" (in a measure sense) regular set is stochastically $C^{2}$. This turns out to be sufficient for the application in $\S 4$.

From now on we assume that

$$
E^{\times}\left[\int_{0}^{\infty}\left|f\left(X_{t}^{(w)}\right)\right| d t\right]<\infty
$$

for all $x$ and all $w \in W$.

LEMMA 3.2. Suppose $\phi(t, u, z)$ is a stochastically $C^{2}$ function in $D=\mathbf{R}^{n+1}$ with respect to $Y_{t}$ satisfying the three conditions

$$
\phi\left(t, U_{t}, z\right) \rightarrow 0 \quad \text { as } t \rightarrow \infty \quad \text { a.s. } P^{y} \quad \text { for all } z \in \mathcal{Z} ; y=(t, u, z),
$$

the family

$$
\left\{\phi\left(\tau, U_{\tau}, z\right)\right\}_{\tau \in T}
$$

is uniformly integrable w.r.t. $P^{y}$ for all $z \in \mathcal{Z}$, where $\mathcal{T}$ is the set of all $\mathcal{F}_{t}$-stopping times and

$$
A \phi+f \leq 0 \text { a.e. with respect to } G(y, \cdot)
$$

Let $t \leq \theta \leq \theta^{\prime} \leq \infty$ be two stopping times. Then for all $y=(t, u, z)$ and all $z_{0}$ we have

$$
\phi\left(\theta, U_{\theta}, z_{0}\right) \geq E^{y}\left[\int_{\theta}^{\theta^{\prime}} f\left(Y_{s}\right) d s+\phi\left(\theta^{\prime}, U_{\theta^{\prime}}, z_{0}\right) \mid \mathcal{F}_{\theta}\right] .
$$

(We interpret $\phi\left(\tau, U_{\tau}, z_{0}\right)$ as 0 if $\tau=\infty\left(\tau=\theta\right.$ or $\left.\theta^{\prime}\right)$ ).

Remark. The stochastically $C^{2}$ requirement corresponds to the "high contact" condition in optimal stopping. See [BØ].

Proof. Choose a constant $T<\infty$ and apply the generalized Dynkin formula (3.3) to $g(t, u, z)=\phi(t, u, z)$ :

$$
\begin{aligned}
& E^{y}\left[\phi\left(\theta^{\prime} \wedge T, U_{\theta^{\wedge} \wedge T}, z\right) \mid \mathcal{F}_{\theta}\right]=\phi\left(\theta \wedge T, U_{\theta \wedge T}, z\right) \\
& +E^{y}\left[\int_{\theta \wedge T}^{\theta^{\prime} \wedge T} A \phi\left(s, U_{s}, z\right) d s \mid \mathcal{F}_{\theta}\right]
\end{aligned}
$$

By (3.8) this gives

$$
\phi\left(\theta \wedge T, U_{\theta \wedge T}, z\right) \geq E^{y}\left[\int_{\theta \wedge T}^{\theta^{\prime} \wedge T} f\left(Y_{s}\right) d s+\phi\left(\theta^{\prime} \wedge T, U_{\theta^{\prime} \wedge T}, z\right) \mid \mathcal{F}_{\theta}\right]
$$

Letting $T \rightarrow \infty$ and using (3.6) and (3.7) we get Lemma 3.2. 
Define the switching operator $M$ on the family $\mathcal{H}$ of Borel measurable functions on $\mathrm{R}^{n+1} \times \mathcal{Z}$ by

$$
M h(t, u, z)=\max _{\zeta \in \mathcal{Z} \backslash\{z\}}\{h(t, u, \zeta)-H(x, \zeta)\} \quad ; h \in \mathcal{H}, \quad x=(t, u, z)
$$

where $H$ is the switching cost function (see (2.7)-(2.9)).

Note that

$$
h(t, u, z) \geq M h(t, u, z) \Leftrightarrow h(t, u, z) \geq h(t, u, \zeta)-H(x, \zeta) \quad \text { for all } \zeta \neq z .
$$

We are now ready for the first main result of this paper.

THEOREM 3.3. Let $\phi$ be a stochastically $C^{2}$ function satisfying (3.6), (3.7), (3.8), as well as the condition

$$
\phi \geq M \phi \text { everywhere }
$$

Then

$$
\phi(x) \geq J^{w}(x) \text { for all } w \in W \text { and all } x .
$$

Proof. Let $w=\left(\theta_{1}, \theta_{2}, \cdots\right)$ with $\theta_{1} \geq t$, let $X_{t}=X_{t}^{(w)}=\left(t, U_{t}, Z_{t}\right)$ and put $\theta_{0}=t$. Since $\phi \geq M \phi$ we get by (3.11) and Lemma 3.2 applied to $\theta=\theta_{k}, \theta^{\prime}=\theta_{k+1}$ and $z_{0}=Z_{\theta_{k}} ; k=$ $0,1,2, \cdots$ :

$$
\begin{aligned}
\phi\left(\theta_{k}, U_{\theta_{k}}, Z_{\theta_{k}}\right) & \geq E^{y}\left[\int_{\theta_{k}}^{\theta_{k+1}} f\left(X_{s}\right) d s+\phi\left(\theta_{k+1}, U_{\theta_{k+1}}, Z_{\theta_{k}}\right) \mid \mathcal{F}_{\theta}\right] \\
& \geq E^{y}\left[\int_{\theta_{k}}^{\theta_{k+1}} f\left(X_{s}\right) d s+\phi\left(\theta_{k+1}, U_{\theta_{k+1}}, \zeta_{k+1}\right)-H\left(X_{\theta_{k+1}}, \zeta_{k+1}\right) \mid \mathcal{F}_{\theta}\right]
\end{aligned}
$$

Now $\zeta_{k+1}=Z_{\theta_{k+1}}$ by (2.5) so if we take expectation and sum from $k=0$ to $k=n-1$ we get, with $y=(t, u, z)$,

$$
\begin{aligned}
& \phi(t, u, z)+\sum_{k=1}^{n-1} E^{y}\left[\phi\left(\theta_{k}, U_{\theta_{k}}, Z_{\theta_{k}}\right)\right] \\
& \geq E^{y}\left[\int_{t}^{\theta_{n}} f\left(X_{s}\right) d s-\sum_{k=1}^{n} H\left(X_{\theta_{k}^{-}}, Z_{\theta_{k}}\right)+\sum_{k=1}^{n} \phi\left(\theta_{k}, U_{\theta_{k}}, Z_{\theta_{k}}\right)\right]
\end{aligned}
$$

Hence

$$
\phi(t, u, z) \geq E^{y}\left[\int_{t}^{\theta_{n}} f\left(X_{s}\right) d s-\sum_{k=1}^{n} H\left(X_{\theta_{k}^{-}}, Z_{\theta_{k}}\right)+\phi\left(\theta_{n}, U_{\theta_{n}}, Z_{\theta_{n}}\right)\right]
$$

Now let $n \rightarrow \infty$. Then $\theta_{n} \rightarrow \infty$ and by (3.6) and (3.7) we get

$$
\phi(t, u, z) \geq E^{y}\left[\int_{t}^{\infty} f\left(X_{s}\right) d s+\sum_{k=1}^{\infty} H\left(X_{\theta_{k}^{-}}, Z_{\theta_{k}}\right)\right]
$$


which is (3.13).

Next we find an optimal impulse control and the corresponding minimal expected cost:

THEOREM 3.4. Suppose $\hat{\phi}=\hat{\phi}(t, u, z)$ is a stochastically $C^{2}$ function satisfying (3.6), (3.7), (3.8), (3.12) and in addition that

$$
A \hat{\phi}+f=0 \quad \text { on } \quad\{(t, u, z) ; \hat{\phi}(t, u, z)>M \hat{\phi}(t, u, z)\}
$$

Define the impulse control $\hat{w}=\left(\hat{\theta}_{1}, \hat{\theta}_{2}, \cdots ; \hat{\zeta}_{1}, \hat{\zeta}_{2}, \cdots\right)$ as follows: Put

$$
\hat{\theta}_{1}=\inf \left\{t>0 ; \hat{\phi}\left(X_{t}^{(0)}\right)=M \hat{\phi}\left(X_{t}^{(0)}\right)\right\}, \quad \text { where } X_{t}^{0}=Y_{t}
$$

and choose $\hat{\zeta}_{1}$ such that

$$
M \hat{\phi}\left(X_{\hat{\theta}_{1}}^{(0)}\right)=\hat{\phi}\left(\hat{\theta}_{1}, U_{\hat{\theta}_{1}}, \hat{\zeta}_{1}\right)-H\left(X_{\hat{\theta}_{1}}^{(0)}, \hat{\zeta}_{1}\right)
$$

Define

$$
Z_{t}^{(1)}= \begin{cases}z & \text { if } 0 \leq t<\hat{\theta}_{1} \\ \hat{\zeta}_{1} & \text { if } \hat{\theta}_{1} \leq t\end{cases}
$$

and put

$$
d X_{t}^{(1)}=\left[\begin{array}{c}
d t \\
d U_{t} \\
d Z_{t}^{(1)}
\end{array}\right]
$$

i.e. $X_{t}^{(1)}$ is the result of applying the impulse control $\hat{w}_{1}=\left(\hat{\theta}_{1}, \infty ; \hat{\zeta}_{1}\right)$ to $Y_{t}$.

Inductively, if stopping times $0 \leq \hat{\theta}_{1} \leq \hat{\theta}_{2} \leq \cdots \leq \hat{\theta}_{k}$ with corresponding impulses $\hat{\zeta}_{1}, \cdots, \hat{\zeta}_{k}$ have been constructed define

$$
\hat{\theta}_{k+1}=\inf \left\{t>\hat{\theta}_{k} ; \hat{\phi}\left(X_{t}^{(k)}\right)=M \hat{\phi}\left(X_{t}^{(k)}\right)\right\}, \quad k \geq 0
$$

where for $k \geq 1 X_{t}^{(k)}$ is the result of applying the impulse control $\hat{w}_{k}=\left(\hat{\theta}_{1}, \cdots, \hat{\theta}_{k}, \infty\right)$ to $Y_{t}$. Next choose $\hat{\zeta}_{k+1}$ such that

$$
M \hat{\phi}\left(\hat{\theta}_{k+1}, U_{\hat{\theta}_{k+1}}, \hat{\zeta}_{k}\right)=\hat{\phi}\left(\hat{\theta}_{k+1}, U_{\hat{\theta}_{k+1}}, \hat{\zeta}_{k+1}\right)-H\left(X_{\hat{\theta}_{k+1}}^{(k)}, \hat{\zeta}_{k+1}\right)
$$

Then $\hat{w} \in W$ and

$$
\hat{\phi}(x)=J^{\hat{w}}(x)
$$

$\hat{w} \quad$ is optimal for Problem 2.1

and

$$
\hat{\phi}=\tilde{\phi} .
$$

Proof. We repeat the arguments of the proofs of Lemma 3.2 and Theorem 3.3. First note that between $\hat{\theta}_{k}$ and $\hat{\theta}_{k+1}$ we have $A \hat{\phi}=-f$ so we get equality if we apply Lemma 3.2 to 
$\theta_{k}=\hat{\theta}_{k}, \theta_{k+1}=\hat{\theta}_{k+1}$ and $\phi=\hat{\phi}$. Therefore we get equality in (3.15) so that for all $n$ we have

$$
\hat{\phi}(t, u, z)=E^{y}\left[\int_{t}^{\hat{\theta}_{n}} f\left(X_{s}\right) d s-\sum_{k=1}^{n} H\left(X_{\hat{\theta}_{k}^{-}}, Z_{\hat{\theta}_{k}}\right)+\hat{\phi}\left(\hat{\theta}_{n}, U_{\hat{\theta}_{n}}, Z_{\hat{\theta}_{n}}\right)\right]
$$

Put $F=\left\{\omega ; \lim _{n \rightarrow \infty} \hat{\theta}_{n}(\omega)<\infty\right\}$ and $\hat{\theta}=\lim _{n \rightarrow \infty} \hat{\theta}_{n}$. Then letting $n \rightarrow \infty$ in (3.25) we get

$$
\hat{\phi}(t, u, z)=E^{y}\left[\int_{t}^{\hat{\theta}} f\left(X_{s}\right) d s-\sum_{k=1}^{\infty} H\left(X_{\hat{\theta}_{k}^{-}}, Z_{\hat{\theta}_{k}}\right)+\hat{\phi}\left(\hat{\theta}, U_{\hat{\theta}}, Z_{\hat{\theta}}\right) \cdot \chi_{F}\right]
$$

But if $\omega \in F$ we have that $\left(\hat{\theta}_{k}, U_{\hat{\theta}_{k}}\right) \rightarrow\left(\hat{\theta}, U_{\hat{\theta}}\right)$ and therefore by $(2.9)$ there exists $a(\omega)>0$ such that

$$
H\left(X_{\hat{\theta}_{k}^{-}}, Z_{\hat{\theta}_{k}}\right) \geq a(\omega) \text { for } \omega \in F \quad \text { for all } k,
$$

which gives

$$
\sum_{k=1}^{\infty} H\left(X_{\tilde{\theta}_{k}^{-}}, Z_{\hat{\theta}_{k}}\right)=\infty \quad \text { for } \omega \in F
$$

From (3.26) and (3.5) we can conclude that $P^{x}(F)=0$, which shows that $\hat{\theta}_{n} \rightarrow \infty$ a.s. Therefore $\hat{w} \in W$.

Now we can apply Theorem 3.3 to $\phi=\hat{\phi}, w=\hat{w}$ and by condition (3.17) we get equality in (3.13). So

$$
\hat{\phi}(x)=J^{\hat{w}}(x)
$$

while from Theorem 3.3

$$
\hat{\phi}(x) \geq J^{w}(x) \text { for all } w \in W .
$$

It follows that $\hat{w}$ is optimal and that

$$
\hat{\phi}(x)=\sup _{w \in W} J^{w}(x)=\tilde{\phi}(x), \quad \text { as claimed. }
$$

Remark. Note that $\hat{\theta}_{k}$ is the first exit time after $\hat{\theta}_{k-1}$ for $X_{t}^{(\hat{w})}$ from the set

$$
D=\{x ; \hat{\phi}(x)>M \hat{\phi}(x)\} \text {. }
$$

Therefore, writing $X_{t}=X_{t}^{(\hat{w})}$, we have

$$
\begin{aligned}
\hat{\phi}\left(X_{\hat{\theta}_{k}}\right) & =M \hat{\phi}\left(X_{\hat{\theta}_{k}^{-}}\right)+H\left(X_{\hat{\theta}_{k}^{-}}, \hat{\zeta}_{k}\right) \\
& =\hat{\phi}\left(X_{\hat{\theta}_{k}^{-}}\right)+H\left(X_{\hat{\theta}_{k}^{-}}^{(k)}, \hat{\zeta}_{k}\right) .
\end{aligned}
$$

Suppose we have strict inequality in (2.8), i.e.

$$
H\left(t, u, z, \zeta_{2}\right)<H\left(t, u, z, \zeta_{1}\right)+H\left(t, u, \zeta_{1}, \zeta_{2}\right) \text { if } z \neq \zeta_{1} \neq \zeta_{2} \neq z .
$$


Then if $\hat{\zeta}_{k-1} \neq \zeta \neq \hat{\zeta}_{k}$ we have

$$
\begin{aligned}
\hat{\phi}\left(X_{\hat{\theta}_{k}}\right) & \geq \hat{\phi}\left(\hat{\theta}_{k}, U_{\hat{\theta}_{k}}, \zeta\right)-H\left(X_{\hat{\theta}_{k^{-}}}, \zeta\right)+H\left(X_{\hat{\theta}_{k}}, \hat{\zeta}_{k}\right) \\
& =\hat{\phi}\left(\hat{\theta}_{k}, U_{\hat{\theta}_{k}}, \zeta\right)-H\left(X_{\hat{\theta}_{k}}, \zeta\right) \\
& +\left[H\left(\hat{\theta}_{k}, U_{\hat{\theta}_{k}}, \hat{\zeta}_{k-1}, \hat{\zeta}_{k}\right)+H\left(\hat{\theta}_{k}, U_{\hat{\theta}_{k}}, \hat{\zeta}_{k}, \zeta\right)-H\left(\hat{\theta}_{k}, U_{\hat{\theta}_{k}}, \hat{\zeta}_{k-1}, \zeta\right)\right. \\
& >\hat{\phi}\left(\hat{\theta}_{k}, U_{\hat{\theta}_{k}}, \zeta\right)-H\left(X_{\hat{\theta}_{k}}, \zeta\right), \quad \text { by }(2.8)^{\prime} .
\end{aligned}
$$

Moreover, by (3.28) we have

$$
\begin{aligned}
\hat{\phi}\left(\hat{\theta}_{k}, U_{\hat{\theta}_{k}}, \hat{\zeta}_{k}\right) & =\hat{\phi}\left(\hat{\theta}_{k}, U_{\hat{\theta}_{k}}, \hat{\zeta}_{k-1}\right)+H\left(\hat{\theta}_{k}, U_{\hat{\theta}_{k}}, \hat{\zeta}_{k-1}, \hat{\zeta}_{k}\right) \\
& >\hat{\phi}\left(\hat{\theta}_{k}, U_{\hat{\theta}_{k}}, \hat{\zeta}_{k-1}\right)-H\left(\hat{\theta}_{k}, U_{\hat{\theta}_{k}}, \hat{\zeta}_{k}, \hat{\zeta}_{k-1}\right)
\end{aligned}
$$

Combining (3.29) and (3.30) we get

$$
\hat{\phi}\left(X_{\hat{\theta}_{k}}\right)>M \hat{\phi}\left(X_{\hat{\theta}_{k}}\right) .
$$

So we see that if (2.8)' holds then the new impulse $\hat{\zeta}_{k}$ brings the state $X_{t}$ back into $D$ at the instant $\hat{\theta}_{k}$ when $X_{t}$ first hits $\partial D$ (the boundary of $D$ ) after $\hat{\theta}_{k-1}$. Thus the optimal strategy can be illustrated as below

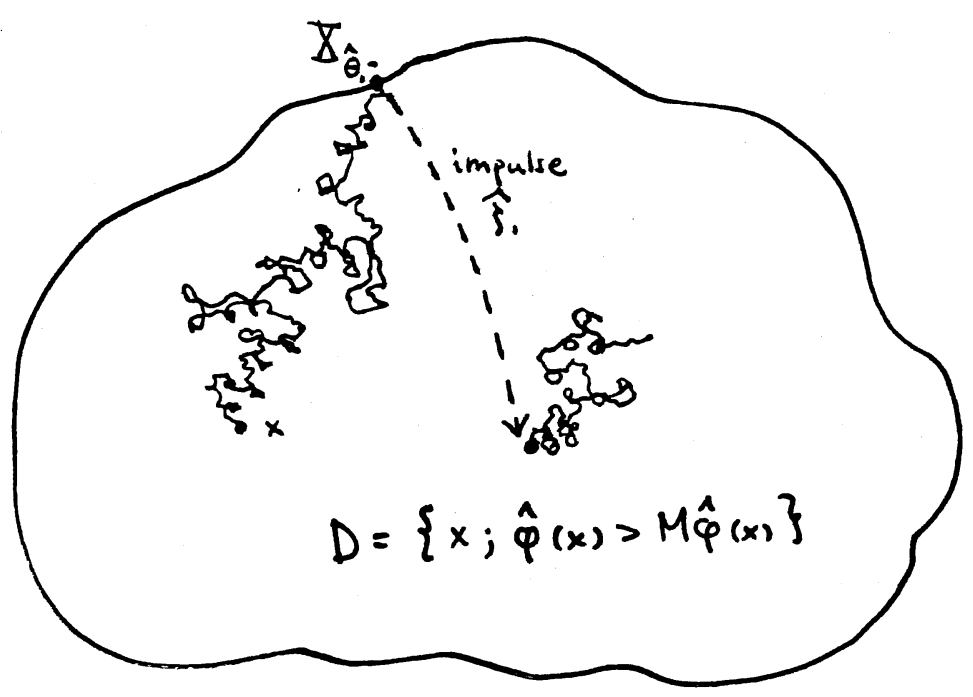

\section{§4. Application to resource extraction}

We assume that the price $P_{t}$ at time $t$ per unit of the resource follows a geometric Brownian motion. This means that $P_{t}$ is the solution of a stochastic differential equation of the form

$$
d P_{t}=\alpha P_{t} d t+\beta P_{t} d B_{t}
$$


where $\alpha, \beta$ are constants and $B_{t}$ is a 1-dimensional Brownian motion. The solution $P_{t}$ of (4.1) is

$$
P_{t}=P_{0} \exp \left(\left(\alpha-\frac{1}{2} \beta^{2}\right) t+\beta B_{t}\right) \quad ; t \geq 0
$$

Let $Q_{t}$ denote the stock of remaining resources in the field. We assume that when the field is open, extraction rate is proportional to the amount of remaining reserves. In other words,

$$
d Q_{t}=-\lambda Z_{t} Q_{t} d t
$$

where $\lambda>0$ is a constant and

$$
Z_{t}= \begin{cases}1 & \text { if the field is open at time } t \\ 0 & \text { if the field is closed at time } t\end{cases}
$$

The state $X_{t}$ of the system at time $t$ is characterized by the 4 quantities $t, P_{t}, Q_{t}, Z_{t}$ :

$$
X_{t}=\left[\begin{array}{c}
t \\
P_{t} \\
Q_{t} \\
Z_{t}
\end{array}\right]
$$

If there is a constant running cost $K>0$ per time unit, the net discounted profit rate $f$ is given by

$$
f(x)=f(t, p, q, z)=(\lambda p q-K) z e^{-\rho t}
$$

So in this case Problem 2.1 becomes

$$
\tilde{\phi}(x)=\sup _{w \in W}\left\{E^{x}\left[\int_{t}^{\infty}\left(\lambda P_{s} Q_{s}-K\right) Z_{s} e^{-\rho s} d s-\sum_{j} H\left(X_{\theta_{j}^{-}}, 1-Z_{\theta_{j}^{-}}\right)\right]\right\},
$$

where $H$ is given by (2.10).

REMARK. It is natural to ask if a better performance could be obtained if - instead of either having the field open at full production or entirely closed - we allow the field to be partially open at all times. If we assume that we can avoid opening and closing costs this way, but have the same running cost $K$, the problem can be formulated as a stochastic control problem as follows:

$$
\Phi(x)=\sup _{\mathcal{H}} E^{x}\left[\int_{t}^{\infty}\left(\lambda \mu_{s} P_{s} Q_{s}-K\right) e^{-\rho s} d s\right]
$$

where $\mu_{t}=\mu\left(X_{t}\right) \in(0,1)$ represents the degree of production (fraction of full production) we choose at state $X_{t}$ and where

$$
d Q_{t}=-\lambda \mu_{t} Q_{t} d t
$$


while $P_{t}$ is as before. The Hamilton-Jacobi-Bellmann equation for this problem states that (see e.g. [Ø, Ch. 11])

$$
\sup _{m \in(0,1)}\left\{(\lambda m p q-K) e^{-\rho t}+\frac{\partial \Phi}{\partial t}+\alpha p \frac{\partial \Phi}{\partial p}+\frac{1}{2} \beta^{2} p^{2} \frac{\partial^{2} \Phi}{\partial p^{2}}-\lambda m q \frac{\partial \Phi}{\partial q}\right\}=0
$$

and that an optimal choice of $\mu$ (if it exists) is a value of $m$ for which the supremum is attained. However, in this case the expression is affine in $m$, so it is clear that no such $m \in(0,1)$ exists. This indicates that the optimal production is "bang-bang": Either full production or no production at all. Therefore it suffices to consider the sequential stopping problem (4.7).

Using the 'high contact principle' it is proved in [BØ] that a solution $\phi_{0}(x)=\phi_{0}(t, p, q, z)$ of the dynamic programming equation (2.12) corresponding to (4.7) is given by

$$
\phi_{0}(t, p, q, z)=e^{-\rho t} \psi_{0}(p, q, z)
$$

where

$$
\psi_{0}(p, q, z)= \begin{cases}u(p, q)-L & \text { if } z=0 \& p \geq \xi \\ z u(p, q)+(1-z) v(p, q) & \text { if } z=0 \& p<\xi \\ & \text { or } z=1 \& p>\eta \\ v(p, q)-C & \text { if } z=1 \& p \leq \frac{\eta}{q}\end{cases}
$$

Here

$$
u(p, q)=\frac{p q}{\rho+\lambda-\alpha}-\frac{K}{\rho}+k_{1}(p q)^{\nu}
$$

and

$$
v(p, q)=k_{2}(p q)^{\gamma}
$$

with

$$
\begin{aligned}
& \gamma=\beta^{-2}\left[-\alpha+\frac{1}{2} \beta^{2}+\sqrt{\left(\alpha-\frac{1}{2} \beta^{2}\right)^{2}+2 \rho \beta^{2}}\right]>1 \text { if } \rho>\alpha \\
& \nu=\beta^{-2}\left[-\alpha+\lambda+\frac{1}{2} \beta^{2}-\sqrt{\left.\left(\alpha-\lambda-\frac{1}{2} \beta^{2}\right)^{2}+2 \rho \beta^{2}\right]}<0\right.
\end{aligned}
$$

and $k_{1}, k_{2}, \xi>\eta>0$ are constants which solve the following system of equations (4.14)(4.17):

$$
\begin{gathered}
\frac{\xi}{\rho+\lambda-\alpha}+k_{1} \xi^{\nu}=k_{2} \xi^{\gamma}+\frac{K}{\rho}+J \\
\frac{\xi}{\rho+\lambda-\alpha}+\nu k_{1} \xi^{\nu}=\gamma k_{2} \xi^{\gamma}
\end{gathered}
$$




$$
\frac{\eta}{\rho+\lambda-\alpha}+k_{1} \eta^{\nu}=k_{2} \eta^{\gamma}+\frac{K}{\rho}-C
$$

$$
\frac{\eta}{\rho+\lambda-\alpha}+\nu k_{1} \eta^{\nu}=\gamma k_{2} \eta^{\gamma}
$$

It is proved in $[B \varnothing]$ that if we assume that

$$
\rho>\alpha
$$

and that

the system (4.14)-(4.17) has a solution $k_{1}, k_{2}, \xi>\eta>0$

then (2.12) holds for $\phi_{0}$, i.e.

$$
\phi_{0}(x)=\sup _{\tau} E^{\alpha}\left[\int_{t}^{\tau}\left(\lambda P_{s} Q_{s}-K\right) Z_{s} e^{-\rho s} d s-H\left(X_{\tau^{-}}, 1-Z_{\tau^{-}}\right)+\phi_{0}\left(X_{\tau}\right)\right]
$$

However, as pointed out earlier this does not imply that $\phi_{0}=\tilde{\phi}$, because it is not clear if the solution of (2.12) is unique.

The strategy $w \in W$ corresponding to the candidate $\phi_{0}$ can be described as follows (see $\S 3)$ :

$$
\left\{\begin{array}{l}
\text { Jump from the } z=0 \text { level (closed field) to the } z=1 \text { level } \\
\text { (open field) as soon as } P_{t} Q_{t} \geq \xi \text { and jump from } z=1 \text { to } z=0 \\
\text { as soon as } P_{t} Q_{t} \leq \eta
\end{array}\right.
$$

Put

$$
H_{0}(z, \zeta)= \begin{cases}L & \text { if } z=0, \zeta=1 \\ C & \text { if } z=1, \zeta=0\end{cases}
$$

Then

$$
H(t, u, z, \zeta)=e^{-\rho t} H_{0}(z, \zeta)
$$

From (4.9) we see that

$$
\begin{array}{lll}
\psi_{0}(p, q, 0)=\psi_{0}(p, q, 1)-H_{0}(0,1) & \text { if } & p \geq \frac{\xi}{q}\left(\Rightarrow p \geq \frac{\eta}{q}\right) \\
\psi_{0}(p, q, 1)=\psi_{0}(p, q, 0)-H_{0}(1,0) & \text { if } & p \leq \frac{\eta}{q}\left(\Rightarrow p \leq \frac{\xi}{q}\right)
\end{array}
$$

and for other values of $(p, q, z)$ we have

$$
\psi_{0}(p, q, z)>\psi_{0}(p, q, 1-z)-H_{0}(z, 1-z) .
$$




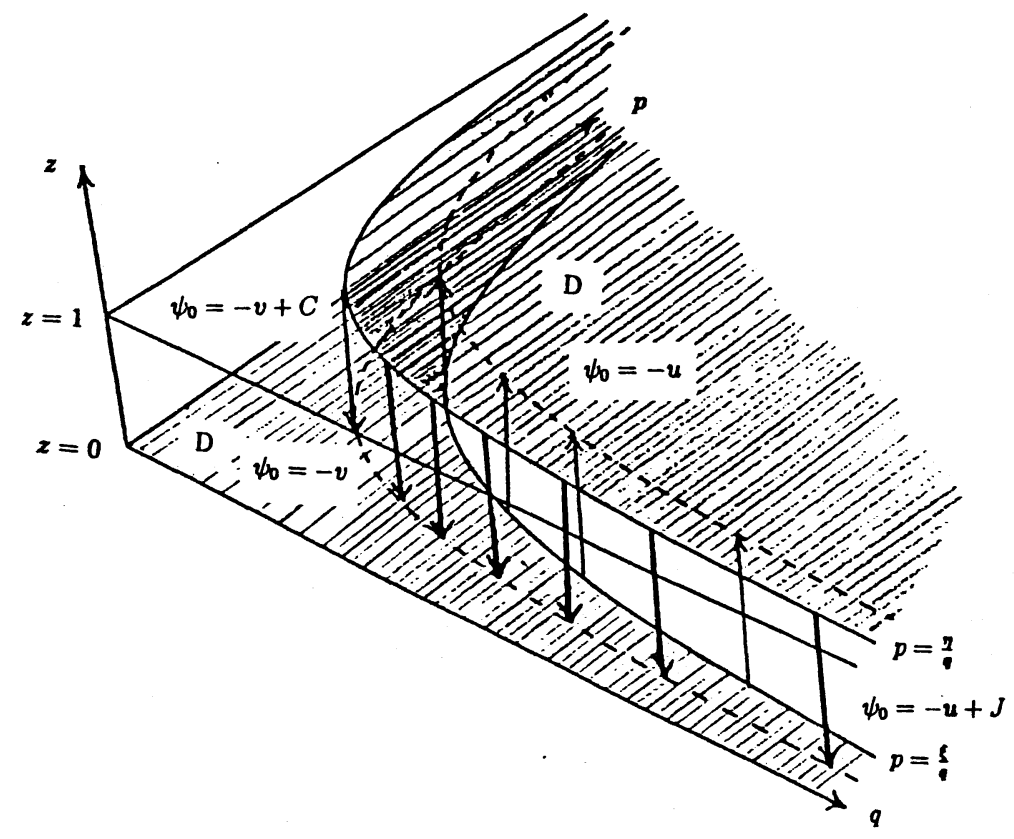

We conclude that, with $M$ defined as in (3.10),

$$
\psi_{0}(p, q, z) \geq M \psi_{0}(p, q, z) \text { for all }(p, q, z)
$$

and if we define the continuation region

$$
D=\left\{(p, q, z) ; z=0 \quad \& \quad p<\frac{\xi}{q} \quad \text { or } \quad z=1 \quad \& \quad p>\frac{\eta}{q}\right\}
$$

then

$$
(p, q, z) \in D \Leftrightarrow \psi_{0}(p, q, z)>M \psi_{0}(p, q, z) .
$$

It follows from [ $\mathrm{B} \emptyset$, Lemma 1$]$ that

$$
\phi_{0} \text { is stochastically } C^{2} \text { with respect to } Y_{t}
$$

In this case the generator $A$ of $Y_{t}$ in (3.2) gets the form

$$
A g(t, p, q)=\frac{\partial g}{\partial t}+\alpha p \frac{\partial g}{\partial p}-\lambda q \frac{\partial g}{\partial q}+\frac{1}{2} \beta^{2} p^{2} \frac{\partial^{2} g}{\partial p^{2}}
$$

In particular, if $g(t, p, q)=e^{-\rho t} h(p, q)$ then

$$
A g=e^{-\rho t} A_{0} h,
$$

where

$$
A_{0} h(p, q)=-\rho h+\alpha p \frac{\partial h}{\partial p}-\lambda q \frac{\partial h}{\partial q}+\frac{1}{2} \beta^{2} p^{2} \frac{\partial^{2} h}{\partial p^{2}}
$$

We now claim that

$$
A_{0} \psi_{0}+f_{0}=0 \text { in } D
$$


and, interpreting $A_{0}$ in the a.e. $G(y, \cdot)$ sense (as with $A$ above),

$$
A_{0} \psi_{0}+f_{0} \leq 0 \text { a.e. in } \mathbf{R}^{+} \times \mathbf{R}^{+} \times\{0,1\}
$$

where $f_{0}=(\lambda p q-K) z$ i.e. $f_{0}=e^{\rho t} f$.

Remark. Let $D_{1}=\{(p, q) ;(p, q, 1) \in D\}$ and $D_{0}=\{(p, q) ;(p, q, 0) \in D\}$. Then by (4.24) we have $D_{1} \cup D_{0}=\mathbf{R}^{+} \times \mathbf{R}^{+}$. So for all $(p, q)$ we have from (4.29) that $A_{0} \psi_{0}(p, q)+f_{0}(p, q, z)=0$ for some $z \in\{0,1\}$. The requirement (4.30) can thus be written

$$
f_{0}(p, q, z) \leq f_{0}(p, q, 1-z) \text { outside } D,
$$

i.e. if we are switching state $z$, we must switch to a state with greater profit rate.

Proof of (4.29): Recall that in [BØ] (formulas (75) and (76)) it is proved (and it is easily checked) that

$$
A_{0} v=0 \text { when } \quad z=0
$$

and

$$
A_{0} u=-f_{0} \text { when } z=1 .
$$

From (4.31) and (4.9) we conclude that

$$
A_{0} \psi_{0}=A_{0} v=0\left(=-f_{0}\right) \quad \text { when } \quad z=0 \& p<\frac{\xi}{q}
$$

Similarly, from (4.32) and (4.9) we get

$$
A_{0} \psi_{0}=A_{0} u=-f_{0} \text { when } z=1 \& p>\frac{\eta}{q}
$$

(4.29) follows from (4.33) and (4.34).

Proof of (4.30): (4.30) is a consequence of (4.20):

The general theory of optimal stopping (see e.g. [Ø]) gives that the right hand side of (4.20) - and hence $\phi_{0}$ itself - is superharmonic with respect to the operator $g \rightarrow A_{0} g+f_{0}$. This implies that $A \phi_{0}+f_{0} \leq 0$ outside $\partial D$ and hence a.e. with respect to $G(y, \cdot)$.

Next we give a condition which ensures that $f$ satisfies (3.5):

LEMMA 4.1. Assume that

$$
\rho>\alpha .
$$

Then

$$
E^{x}\left[\int_{0}^{\infty}\left|f\left(X_{t}^{(w)}\right)\right| d t\right]<\infty \quad \text { for all } \quad w \in W
$$


Proof. Since $Z_{t} \leq 1$ and $Q_{t} \leq Q_{0}$ for all $t$ and all $w$ it suffices to prove that

$$
E^{y}\left[\int_{0}^{\infty} P_{t} e^{-\rho t} d t\right]<\infty \text { for } y=(0, p, q, 1)
$$

Now

$$
P_{t}=p \cdot \exp \left(\left(\alpha-\frac{1}{2} \beta^{2}\right) t+\beta B_{t}\right)
$$

Hence

$$
E^{y}\left[\int_{0}^{\infty} P_{t} e^{-\rho t} d t\right]=\int_{0}^{\infty} \exp ((\alpha-\rho) t) d t<\infty
$$

since $\alpha-\rho<0$.

Finally we observe from (4.8) and (4.9) that the function $\phi_{0}=e^{-\rho t} \psi_{0}$ satisfies (3.6). To verify (3.7) choose $\epsilon>0$ and consider

$$
R_{t}=P_{t}^{1+\epsilon} e^{-\rho(1+\epsilon) t}=p^{1+\epsilon} \cdot \exp \left(\left(\alpha-\frac{1}{2} \beta^{2}-\rho\right)(1+\epsilon) t+\beta(1+\epsilon) B_{t}\right)
$$

Note that

$$
R_{t}=R_{0}+\int_{0}^{t} \gamma R_{s} d s+\int_{0}^{t} \sigma R_{s} d B_{s}
$$

where

$$
\sigma=\beta(1+\epsilon)
$$

and

$$
\gamma=\left(\alpha-\frac{1}{2} \beta^{2}-\rho\right)(1+\epsilon)+\frac{1}{2} \beta^{2}(1+\epsilon)^{2}=(\alpha-\rho)+\epsilon\left(\alpha+\frac{1}{2} \beta^{2}-\rho+\frac{1}{2} \beta^{2} \epsilon\right)
$$

Choose $\epsilon>0$ so small that $\gamma<0$ and let $\tau$ be a stopping time. For all natural numbers $N$ we have

$$
\begin{aligned}
& E^{\propto}\left[\left|\phi_{0}\left(\tau \wedge N, U_{\tau \wedge N}, z\right)\right|^{1+\epsilon}\right] \leq q^{1+\epsilon} E^{x}\left[R_{\tau \wedge N}\right] \\
& =(p q)^{1+\epsilon}+E^{x}\left[\int_{0}^{\tau \wedge N} \gamma R_{s} d s\right] \leq(p q)^{1+\epsilon}
\end{aligned}
$$

Letting $N \rightarrow \infty$ we get

$$
E^{x}\left[\left|\phi_{0}\left(\tau, U_{\tau}, z\right)\right|^{1+\epsilon}\right] \leq(p q)^{1+\epsilon}
$$

for all stopping times $\tau$. This implies (3.7).

Summing up we conclude:

THEOREM 4.2. Assume that (4.19) and (4.35) hold. Then the function $\phi_{0}=e^{-\rho t} \psi_{0}$ given by (4.8) and (4.9) solves the starting and stopping problem (4.7).

The corresponding optimal impuls control $\hat{w}$ is given by (4.21). 
Proof. By (4.35) $f$ satisfies condition (3.5). The function $\phi=\phi_{0}$ satisfies conditions (3.6), (3.7), as well as (3.8), (3.12) and (3.17) in virtue of (4.30), (4.22) and (4.29), respectively. Therefore Theorem 3.4 applies to $\phi_{0}$ and the proof is complete.

\section{Acknowledgements.}

We are grateful to Marcus Miller for useful discussions.

\section{REFERENCES}

[BL] A. Bensousson and J.-L. Lions: Impulse Control and Quasi-Variational Inequalities. Gauthier-Villars 1984.

[BØ] K.A. Brekke and B. Øksendal: "The high contact principle as a sufficiency condition for optimal stopping". In D. Lund and B. Øksendal (editors): Stochastic Models and Option Values. North-Holland 1991, pp. 187-208.

[BS] M.J. Brennan and E.S. Schwartz: "Evaluating natural resource investments". Journal of Business 58 (1985), pp. 135-157.

[D] A. Dixit: "Entry and exit decisions under uncertainty". Journal of Political Economy 97 (1989), pp. 620-638.

[MZ] M. Miller and L. Zhang: "Irreversibility and oil production". University of Warwick (to appear).

[Ø] B. Øksendal: "Stochastic Differential Equations" (3 $3^{\text {rd }}$ edition). Springer-Verlag 1992. 